\title{
Do diverse antifreeze protein structures bind ice by the same mechanism?
}

\author{
Qilu Ye, Koli Basu, Robert Eves, Robert L. Campbell, Sean Phippen and Peter L. Davies
}

Department of Biomedical and Molecular Sciences, Queen's University, Kingston, ON

Canada

\begin{abstract}
Antifreeze proteins (AFPs) are characterized by their capacity to adsorb to the surface of ice crystals and prevent their growth. This adsorption lowers the freezing temperature of a solution below its melting point. AFPs have independently evolved in a variety of organisms that may encounter the threat of freezing, including many species of polar fish, insects, plants and microorganisms. Despite their diverse origins and structures we suggest that all AFPs organize ice-like water patterns on one side of the protein (the icebinding site) that then bind the AFP to ice. Here, to help test this hypothesis, we have solved two AFP crystal structures. One is of Lake Ontario midge (Chironomidae) AFP, which has intermediate antifreeze activity. Previously our group modelled the midge AFP based on its sequence characteristics with the crystal structure of Tenebrio molitor AFP as a template. The midge crystal structure at $1.9 \AA$-resolution is a close match to the modelled structure and shows a 10-residue repeated solenoid fold, with 8 disulfide-bonds stabilizing the coils and 7 Tyr pointing outward from one side of the solenoid structure as a potential ice-binding site. The second protein crystal structure is from Rhagium mordax, a longhorn beetle, solved at $2.05-\AA$ resolution. This AFP is hyperactive and its crystal structure resembles that of the Rhagium inquisitor ortholog in having a $\beta$-solenoid fold with a wide, flat, ice-binding surface formed by four parallel rows of mainly Thr residues. The key difference between these structures is that the Rhagium mordax AFP has crystallized with its ice-binding site exposed to solvent providing the opportunity to see the organization of surface waters. In contrast the Rhagium inquisitor AFP crystallized with its ice-binding site making protein-protein contacts that obscure the surface water patterns.

Supported by CIHR and the Canada Research Chair program
\end{abstract}

